\title{
A Fluorescent Probe for TNP Detection in Aqueous Solution Based on Joint Properties of Intramolecular Charge Transfer and Aggregation- Induced Enhanced Emission
}

\author{
Hongwei Ma, $\$$ Chuanyou He, $\$$ Xueliang Li, Obolda Ablikim, Shitong Zhang, Ming Zhang*
}

State Key Laboratory of Supramolecular Structure and Materials, Jilin University, Changchun 130012, P. R. China. Tel: +86-431-85167507. Fax: +86-431-85193421.

$\$$ These authors contributed equally to this work.

Keywords: fluorescent probe; TNP; intramolecular charge transfer; aggregation-induced enhanced emission

\begin{abstract}
A donor-acceptor (D-A) fluorescent molecule, Nph-An composed of anthracene and 1, 8Naphthalimide units, was designed and synthesized. It presented typical intramolecular charge transfer (ICT) emission from 1, 8-Nphthalimide units to anthracene units, and this emission was enhanced obviously in aqueous solution, which was attributed to aggregation-induced enhanced emission (AIEE). Further, Nph-An was used as a fluorescent probe to detect 2, 4, 6-trinitrophenol (TNP) in aqueous solution. The proton transfer from TNP to Nph-An could efficiently block the ICT emission of Nph-An, and cause the fluorescence remarkably to be quenched. The calculated limit detection is $4.7 \times 10^{-7} \mathrm{M}$.
\end{abstract}

\section{Introduction}

Due to the extremely danger of the nitroaromatics explosives in public security, human health and environment, many efforts have been devoted to detecting explosives [1]. Among the 
nitroaromatics explosives, 2, 4, 6-trinitrotoluene (TNT) and 2, 4, 6-trinitrophenol (TNP) are the most widely used in dyes, explosives, fireworks, matches, and pharmaceutical industries and their residues often cause much damages [2]. In particular, TNP has higher solubility in water than TNT, which is easily absorbed by human and animals and causes serious diseases [3]. Thus, the detection of TNP in water is of more importance. However, less attention has been paid to TNP than other explosives [4]. Thereby, it is necessary to develop a rapid, sensitive and portable method to detect TNP, especially in aqueous solution.

Up to now, some materials have been employed to detect TNP, including graphitic carbon nitride nanosheets [5], polydiacetylene microtube [6], metal complex [7], electron-rich polymer [8], and metal-organic framework [9]. Among them, fluorescent materials received more and more attentions, due to their sensitive, simple and low-cost properties [10]. The mechanism of TNP fluorescent detection is mainly ascribed to the photo-induced electron transfer (PET). Though many good results have been achieved for the fluorescent detection of TNP, in our opinion, it still remains a challenge to develop a facile way to fabricate fluorescent probe in aqueous solution.

As we known, the conventional fluorescent materials are commonly aggregated in aqueous solution, and the fluorescence is often weakened or quenched due to the strong intermolecular $\pi-\pi$ interactions. The phenomenon is usually called "aggregation-caused quenching" (ACQ) $[11,12]$. But some fluorescent materials present opposite properties, which have strong emission in high concentration or solid state [13]. It is called aggregation-induced emission or enhanced emission (AIE/AIEE) [14], which offers the opportunity for fluorescent detection in aqueous solution. On the other hand, some donor-acceptor molecules with highly efficient Intramolecular charge transfer (ICT) emission have been reported $[15,16]$. The ICT emission can easily be blocked by TNP, causing the emission remarkably decreased [17], which could afford the possibility to 
detect TNP. Up to date, the reports combining AIEE and ICT to detect TNP in aqueous solution are rare.

In this work, we report a molecule, Nph-An. Its molecular structure is shown in Fig 1a. NphAn presents strong fluorescence emission in aqueous solution. Moreover, Nph-An is a donor (D) acceptor (A) type molecule, composed of 1,8-Naphthalimide units as the acceptor and anthracene units as the donor, respectively. Its emission is attributed to the intramolecular charge transfer (ICT) from 1, 8-Naphthalimide units to anthracene units.

Based on the features of AIEE and ICT emission, Nph-An was used in the fluorescent detection of TNP in aqueous solution with high sensitivity. There exists strong electrostatic interaction between Nph-An and TNP, due to the protonation of Nph-An caused by TNP molecule in aqueous solution [18-19]. The electrostatic interaction could efficiently block the ICT emission from 1, 8-Naphthalimide to anthracene moieties of Nph-An inducing the strong fluorescent quenching.

\section{Experimental}

\subsection{Materials and methods}

All other chemicals and solvents were purchased from commercial sources and used as received except where noted. Reactions were all conducted under argon atmosphere. ${ }^{1} \mathrm{H}$ NMR spectra were recorded on a Bruker AVANCZ 500/600 spectrometer at 500/600 MHz, using $\mathrm{CDCl}_{3} / \mathrm{DMSO}$ as the solvent at $298 \mathrm{~K}$, and tetramethylsilane (TMS) as the internal standard. Ultraviolet-visible (UV-vis) absorption spectra were recorded on a model UV-3100 spectrophotometer. Fluorescence spectra were performed using a model RF-5301PC spectrophotometer. Energy level was calculated by DFT/B3LYP/6-31g (d, p).

\subsection{Synthesis of Nph-An}


Compound 1: A mixture of 4-bromo-N-phenyl-1, 8-naphthalimide (1 mmol), aniline (2 mmol), added into Acetic Acid (10 mL). The mixture was refluxed for $12 \mathrm{~h}$, then the mixture was poured into ice water and filtered. The crude product was recrystallized with ethanol to obtain light grey solid. The yield was $85.3 \%, 281.74 \mathrm{mg} .{ }^{1} \mathrm{H}$ NMR (500 MHz, $\left.\mathrm{CDCl}_{3}\right) \delta 8.74(\mathrm{~d}, \mathrm{~J}=7.3 \mathrm{~Hz}, 1 \mathrm{H})$, $8.68(\mathrm{~d}, \mathrm{~J}=8.5 \mathrm{~Hz}, 1 \mathrm{H}), 8.50(\mathrm{~d}, \mathrm{~J}=7.8 \mathrm{~Hz}, 1 \mathrm{H}), 8.12(\mathrm{~d}, \mathrm{~J}=7.8 \mathrm{~Hz}, 1 \mathrm{H}), 7.93(\mathrm{t}, \mathrm{J}=7.9 \mathrm{~Hz}$ 1H), $7.59(\mathrm{t}, \mathrm{J}=7.6 \mathrm{~Hz}, 2 \mathrm{H}), 7.52(\mathrm{dd}, \mathrm{J}=14.8,7.4 \mathrm{~Hz}, 1 \mathrm{H}), 7.34(\mathrm{dd}, \mathrm{J}=13.2,5.9 \mathrm{~Hz}, 2 \mathrm{H})$. MALDI-TOF-MS: 351.87.

Compound 2: A mixture of 9-bromo-10-(naphthalen-2-yl) anthracene (383.28 mg, $1 \mathrm{mmol}$ ), bis(pinacolato) diboron (380.91 mg, $1.5 \mathrm{mmol}$ ), [1,1'-bis(diphenyl-phosphino) ferrocene] dichloropalladium $(24.50 \mathrm{mg}, 0.03 \mathrm{mmol})$ and potassium acetate $(588.84 \mathrm{mg}, 6 \mathrm{mmol})$ were dissolved in $10 \mathrm{~mL}$ of 1, 4-dioxane. The solution was heated with stirring at $80{ }^{\circ} \mathrm{C}$ for $24 \mathrm{~h}$ under nitrogen. The cooled mixture was washed with water and extracted with DCM. Then the DCM solution was dried with anhydrous magnesium sulfate overnight. After removing the solvent, the obtained crude product was purified by silica gel chromatography using DCM/petroleum ether $(1: 1, \mathrm{v} / \mathrm{v})$ as elute. The yield was $58.2 \%, 250.46 \mathrm{mg} .{ }^{1} \mathrm{H}$ NMR $\left(500 \mathrm{MHz}, \mathrm{CDCl}_{3}\right) \delta 8.49(\mathrm{~d}, \mathrm{~J}=$ $8.8 \mathrm{~Hz}, 2 \mathrm{H}), 8.09-8.02(\mathrm{~m}, 2 \mathrm{H}), 7.93(\mathrm{~d}, \mathrm{~J}=9.0 \mathrm{~Hz}, 2 \mathrm{H}), 7.68(\mathrm{~d}, \mathrm{~J}=8.8 \mathrm{~Hz}, 2 \mathrm{H})$, 7.64-7.58 (m, 2H), 7.52 (ddd, J = 15.2, 8.1, 2.1 Hz, 3H), 7.35-7.29 (m, 2H), 1.65 (s, 12H). MALDI-TOF-MS: 575.39.

Nph-An: A mixture of compound 1 (0.75 mmol, $264.14 \mathrm{mg})$, compound 2 (0.5 mmol, 215.18 $\mathrm{mg})$, and tetrakis (triphenylphosphine) palladium(0) $(0.015 \mathrm{mmol}, 17.34 \mathrm{mg})$ was added into a 50 $\mathrm{mL}$ flask. Potassium carbonate $(0.015 \mathrm{mmol}, 0.55 \mathrm{~g})$ was dissolved in $2 \mathrm{~mL}$ water and then the solution was added to the $50 \mathrm{~mL}$ flask. Toluene $(6 \mathrm{~mL})$ was added to the above flask, and the solution was refluxed at $85{ }^{\circ} \mathrm{C}$ for $48 \mathrm{~h}$ under nitrogen. The cooled mixture was quenched with dilute hydrochloric acid solution and extracted with DCM. The organic extracts were dried over anhydrous magnesium sulfate and concentrated by rotary evaporation. The obtained product was 
purified by silica gel chromatography using DCM as elute. The yield was $60.5 \%, 174.13 \mathrm{mg} .{ }^{1} \mathrm{H}$ NMR (600 MHz, DMSO) $\delta 8.78(\mathrm{~d}, \mathrm{~J}=7.3 \mathrm{~Hz}, 1 \mathrm{H}), 8.56(\mathrm{~d}, \mathrm{~J}=7.1 \mathrm{~Hz}, 1 \mathrm{H}), 8.25(\mathrm{t}, \mathrm{J}=7.7 \mathrm{~Hz}$, 1H), 8.19-8.04 (m, 4H), 7.75-7.65 (m, 6H), $7.60(\mathrm{t}, \mathrm{J}=7.6 \mathrm{~Hz}, 2 \mathrm{H}), 7.54-7.38(\mathrm{~m}, 8 \mathrm{H}), 7.33(\mathrm{~d}, \mathrm{~J}$ $=8.8 \mathrm{~Hz}, 2 \mathrm{H})$. MALDI-TOF-MS: 575.12.

\subsection{Fluorescent Detection of TNP and other explosives in aqueous solution}

Nph-An was resolved in the mixture solvent (water/THF=9/1) with a concentration of $2 \mu \mathrm{M}$. Firstly, $3 \mathrm{~mL}$ Nph-An solution were put into the cuvette, then adding $3 \mu \mathrm{L}$ of TNP/TNT $(0.01 \mathrm{M})$ into the cuvette and being stirred for $10 \mathrm{~s}$ every time, then the fluorescence spectrum was recorded under UV light at $365 \mathrm{~nm}$.

\section{Results and discussion}

Nph-An was synthesized according to the synthetic route presented as Fig. 1a by Suzuki coupling reaction between compound 1 [20] and compound 2 [21]. Nph-An is composed of 1, 8Naphthalimide units as the acceptor and anthracene units as the donor, respectively. According to the Density Functional Theory (DFT) calculation shown as Fig. 1b, the lowest unoccupied molecular orbital (LUMO) and the highest occupied molecular orbital (HOMO) of Nph-An mainly localized on the 1, 8-Naphthalimide units and anthracene units respectively, which is a typical D-A molecule orbital distribution.

\section{Insert Figure 1}

Fig. 1 (a) Synthetic route of Nph-An; (b) Distribution of HOMO and LUMO of Nph-An calculated by TD-DFT.

The absorption and photoluminescence (PL) spectra of Nph-An are shown in Fig 2a. It can be seen that there are mainly two absorption bands located at $371 \mathrm{~nm}$ and $262 \mathrm{~nm}$, respectively. The absorption band around $371 \mathrm{~nm}$ is assigned to anthracene units [22]. There is one PL band of 
Nph-An in dichloromethane (DCM) peaking at $558 \mathrm{~nm}$. To investigate the ICT properties of Nph-An, the fluorescent spectra in different polarity solvents are tested. As can be seen from Fig $2 \mathrm{~b}$, the peaks of emission spectra changed from $460 \mathrm{~nm}$ in cyclohexane to $586 \mathrm{~nm}$ in DMSO as the solvent polarity increases, which suggests the ICT properties of Nph-An. The color change of Nph-An in different solvents is shown as the inset of Fig. $2 b$.

\section{Insert Figure 2}

Fig. 2 (a) Absorption and PL spectra of Nph-An in DCM; (b) PL spectra of Nph-An recorded in Cyclohexane, Toluene, THF and DMSO, respectively. Inset: the pictures of Nph-An are in different solvents under UV light at $365 \mathrm{~nm}$.

The AIEE behavior of Nph-An was evaluated by adding different water fractions $\left(f_{w}\right)$ into Nph-An THF solution. It can be seen from Fig. 3, the PL intensity changed a lot. When $f_{w}$ increased to $50 \%$, nearly no fluorescence of Nph-An can be observed, which may be attributed to the dipole-dipole interaction between the fluorescent molecule in its excited state and the surrounding solvent molecules [23]. As $\mathrm{f}_{\mathrm{w}}$ is further increased and higher than $50 \%$, the emission intensity is enhanced. When $\mathrm{f}_{\mathrm{w}}=90 \%$, the emission intensity reach its maximum and is as 1.7 times as its original intensity. The generated aggregation decreased the intramolecular nonradiative transition, inducing the enhanced emission.

\section{Insert Figure 3}

Fig. 3 The PL spectra of Nph-An recorded in water/THF mixture solvent with different $f_{w}$. The inset: the change of emission with different $\mathrm{f}_{\mathrm{w}}$.

The fluorescent detection is performed by recording the PL spectra of Nph-An in mixture solvent (water/THF=9/1) upon gradually adding TNP aqueous solution. As Fig. 4a presents, adding TNP aqueous solution to Nph-An mixture solution caused significant decrease of PL 
intensity. The fluorescence quenching efficiency is $88.3 \%$ upon the addition of TNP with the concentration of $1 \times 10^{-4} \mathrm{M}$.

The fluorescent static quenching coefficient $K_{s v}$ is an important factor to evaluate the sensor, which can be calculated according to Stern-Volmer equation:

$$
\frac{I_{0}}{I}=1+K_{s v}[Q]
$$

where $I_{0}$ and $I$ are the fluorescence intensity in the absence and presence of TNP, respectively. $K_{s v}$ is the quenching constant, Q is the TNP concentration. Accordingly, the $K_{s v}$ is calculated to be $7.0 \times 10^{4} \mathrm{M}^{-1}$, and according to the limits of detection (LODs) $=3 \mathrm{~S}_{\mathrm{B}} / \mathrm{m}$ [24], where $\mathrm{S}_{\mathrm{B}}$ is the standard deviation of the blank measurements and $\mathrm{m}$ is the slope of intensity versus sample concentration, the LODs of the Nph-An is calculated to be $4.7 \times 10^{-7} \mathrm{M}$, which is comparable to the values previously reports listed in Table 1 . However, most of the reports listed in Table 1 were obtained in non-aqueous solutions, which limited their practical application. Some reported materials in aqueous solutions listed in Table 1 exhibited relatively high LODs.

\section{Insert Table 1}

Table 1 Some reported results for the detection of TNP in solution.

Further, the selectivity of the fluorescence sensor of Nph-An was tested against TNP, TNT and 2, 4-Dinitrotoluene (DNT). As can be seen from Fig. 4b, compared to TNT and DNT, TNP caused the fluorescence of Nph-An to be remarkably decreased in mixture solution (water/THF=9/1) solution.

\section{Insert Figure 4}


Fig. 4 (a) PL spectra of Nph-An $(2 \mu \mathrm{M})$ upon gradual addition of TNP (from 0 to $1 \times 10^{-4} \mathrm{M}$ ) in mixture solvent (water/THF=9/1). Inset is the Stern-Volmer plots; (b) Different fluorescence quenching efficiency of Nph-An exposed in TNP, TNT and DNT solution, respectively.

Fluorescence resonance energy transfer could lead to the fluorescence quenched. But this pathway can be eliminated because the absorption spectrum of TNP has the little overlap with the PL spectrum of Nph-An (Fig. 5a). The intensity of absorption spectra of Nph-An increased upon the addition of TNP (Fig. 5b), and the solution color changed from colorless to pale yellow indicating the formation of TNP anion [34]. While for the addition of TNT or DNT, no obvious color change was observed.

\section{Insert Figure 5}

Fig. 5 (a) Absorption spectrum of TNP and the PL spectrum of Nph-An recorded in mixture solvent; (b) Absorption spectra of Nph-An solution $(2 \mu \mathrm{M})$ recorded with addition of TNP in THF. The picture is Nph-An solution changed from colorless (without TNP) to pale yellow (with TNP).

${ }^{1} \mathrm{H}$ NMR spectra is an efficient method to clarify the interaction between TNP and Nph-An. With the addition of TNP to Nph-An in $\mathrm{CDCl}_{3}$, the proton signals of ' $\mathrm{a}, \mathrm{b}, \mathrm{q}, \mathrm{r}, \mathrm{p}$, and s' position are upfield shifted $(\Delta \delta=0.01)$ and other protons signals nearly have no shift (Fig. 6). The results imply that TNP molecule could make Nph-An protonated leading to the strong electrostatic interaction between the protonated Nph-An and TNP anion.

\section{Insert Figure 6}

Fig. 6 Schematic illustration of the proposal intermolecular interaction mode and ${ }^{1} \mathrm{H}$ NMR spectra of Nph-An $(10 \mu \mathrm{M})$ is with and without TNP in $\mathrm{CDCl}_{3}$. 
To get further understanding of the mechanism of fluorescence quenching between Nph-An and TNP, theoretical calculations were carried out with Gaussian 09 package under DFT/B3LYP/6-31g (d, p) method depicted in Fig. 7. According to the theoretical calculations of Nph-An, the LUMO (-2.35 eV) and the HOMO (-5.41 eV) of Nph-An mainly localized on the 1, 8-Naphthalimide units and on anthracene units, respectively. However, the orbital was redistributed when Nph-An was protonated by TNP and the electrostatic interaction between the protonated Nph-An and TNP anion appeared. As can be seen, after adding TNP, the LUMO ($3.17 \mathrm{eV}$ ) mainly localized on TNP anion, which favored the electron transfer from the excited state of Nph-An to TNP anion in the fluorescence quenching process. So Nph-An exhibited high fluorescence quench efficiency to TNP in aqueous solution. These results indicate the protonation of carbanyl group by the acidic behavior of TNP blocked the ICT emission from 1, 8Naphthalimide units to anthracene units, which enhanced the fluorescence quenching effect.

\section{Insert Figure 7}

Fig. 7 Calculated energy diagram of Nph-An and the complex of Nph-An and TNP.

We also performed the detection of the TNP residual products using the filter paper. Because inevitably contacting with TNP in actual production, such as preparation and packaging of explosive devices, some TNP residual products will be in human clothes and skins. So a low-cost, simple and fast method to detect TNP is very necessary. A piece of common filter paper was immersed into the Nph-An solution and dried under vacuum. It exhibited strong emission under UV light (Fig. 8b). After a human thumb rubbed with TNP pressed on the filter paper for $20 \mathrm{~s}$. The fingerprint of the thumb is clearly observed under UV light (Fig. 8c).

\section{Insert Figure 8}


Fig.8 (a) Photos of filter paper were immersed in Nph-An solution $(10 \mu \mathrm{M})$ and dried under vacuum. (b) Photos of filter under UV light at $365 \mathrm{~nm}$. (c) Photos of filter paper contact with human thumb, which rubbed with TNP.

\section{Conclusions}

In conclusion, we have synthesized a small fluorescent molecule Nph-An, the structural design integrated intramolecular charge transfer and aggregation-induced enhanced emission. Nph-An was found to be an efficient fluorescent probe for TNP detection in aqueous solution. The protonation of carbanyl group by the acidic behavior of TNP blocked the ICT emission of Nph-An, and caused the fluorescence remarkably to be quenched.

\section{Acknowledgements}

We are grateful for the financial support from National Science Foundation of China (grant numbers 50973041, 21374037) and Graduate Innovation Fund of Jilin University (Project 2015045).

\section{References}

[1] Y. Ma, S. Wang, L. Wang, Nanomaterials for luminescence detection of nitroaromatic explosives, Trends Analyt. Chem. 65 (2015), 13-21.

[2] J. Ye, L. Zhao, R. F. Bogale, Y. Gao, X. Wang, X. Qian, S. Guo, J. Zhao, G. Ning, Highly selective detection of 2, 4, 6-trinitrophenol and $\mathrm{Cu}^{2+}$ ions based on a fluorescent cadmium-pamoate metal-organic framework, Chem. Eur. J. 21 (2015), 2029-2037.

[3] B. Roy, A. K. Bar, B. Gole, P. S. Mukherjee, Fluorescent tris-imidazolium sensors for picric acid explosive, J. Org. Chem. 78 (2013), 1306-1310. 
[4] Y. Peng, A. -J. Zhang, M. Dong, Y. -W. Wang, A colorimetric and fluorescent chemosensor for the detection of an explosive-2, 4, 6-trinitrophenol (TNP), Chem. Commun. 47 (2011), 4505-4507.

[5] M. Rong, L. Lin, X. Song, T. Zhao, Y. Zhong, J. Yan, Y. Wang, X. Chen, A label-free fluorescence sensing approach for selective and sensitive detection of 2, 4, 6-trinitrophenol (TNP) in aqueous solution using graphitic carbon nitride nanosheets, Anal. Chem. 87 (2015), $1288-1296$.

[6] G. Yang, W. Hu, H. Xia, G. Zou, Q. Zhang, Highly selective and reproducible detection of picric acid in aqueous media, based on a polydiacetylene microtube optical waveguide, $\mathrm{J}$. Mater. Chem. A 2 (2014), 15560-15565.

[7] A. Gupta, Y. -A. Kang, M. -S. Choi, J. S. Park, Characteristic response of tetra(methylbenzyloxy)-substituted zinc-phthalocyanine toward picric acid, Sens. Actuat. B: Chem. 209 (2015) 225-229.

[8] L. Bai, P. Wang, P. Bose, P. Li, R. Zou, Y. Zhao, Macroscopic architecture of charge transfer-induced molecular recognition from electron-rich polymer interpenetrated porous frameworks, ACS Appl. Mater. Interfaces 7 (2015), 5056-5060.

[9] J. Ye, X. Wang, R. F. Bogale, L. Zhao, H. Cheng, W. Gong, J. Zhao, G. Ning, A fluorescent zinc-pamoate coordination polymer for highly selective sensing of 2, 4, 6trinitrophenol and $\mathrm{Cu}^{2+}$ ion, Sens. Actuat. B: Chem. 210 (2015) 566-573.

[10] M. E. Germain, M. J. Knapp, Optical explosives detection: from color changes to fluorescence turn-on, Chem. Soc. Rev. 38 (2009), 2543-2555.

[11] J. B. Birks, Photophysics of Aromatic Molecules, Wiley, London, UK, 1970.

[12] J. R. Lakowicz, Principles of Fluorescence Spectroscopy, Springer, Singapore, 2006.

[13] R. Hu, J. L. Maldonado, M. Rodriguez, C. Deng, C. K. W. Jim, J. W. Y. Lam, M. M. F. Yuen, G. Ramos-Ortiz, B. Z. Tang, Luminogenic materials constructed from 
tetraphenylethene building blocks: Synthesis, aggregation-induced emission, two-photon absorption, light refraction, and explosive detection, J. Mater. Chem. 22 (2012), 232-240.

[14] Y. Hong, J. W. Y. Lam, B. Z. Tang, Aggregation-induced emission, Chem. Soc. Rev. 40 (2011), 5361-5388.

[15] J. Li, Y. Qian, L. Xie, Y. Yi, W. Li, W. Huang, From dark TICT state to emissive quasiTICT State: the AIE mechanism of $N$-(3-(benzo[ $d]$ oxazol-2-yl)phenyl)-4-tertbutylbenzamide, J. Phys. Chem. C 119 (2015), 2133-2141.

[16] S. Hirata, Y. Sakai, K. Masui, H. Tanaka, S. Y. Lee, H. Nomura, N. Nakamura, M. Yasumatsu, H. Nakanotani, Q. Zhang, K. Shizu, H. Miyazaki, C. Adachi, Highly efficient blue electroluminescence based on thermally activated delayed fluorescence, Nat. Mater. 14 (2015), 330-336.

[17] Y. Xu, B. Li, W. Li, J. Zhao, S. Sun, Y. Pang, "ICT-not-quenching" near infrared ratiometric fluorescent detection of picric acid in aqueous media, Chem. Commun. 49 (2013), 4764-4766.

[18] A. Ding, L. Yang, Y. Zhang, G. Zhang, L. Kong, X. Zhang, Y. Tian, X. Tao, J. Yang, Complex-formation-enhanced fluorescence quenching effect for efficient detection of picric acid, Chem. Eur. J. 20 (2014), 12215-12222.

[19] M. Kumar, S. I. Reja, V. Bhalla, A charge transfer amplified fluorescent $\mathrm{Hg}^{2+}$ complex for detection of picric acid and construction of logic functions, Org. Lett. 14 (2012), 60846087.

[20] P. A. Panchenko, Y. V. Fedorov, O. A. Fedorova, G. Jonusauskas, Comparative analysis of the PET and ICT sensor properties of 1,8-naphthalimides containing aza-15-crown-5 ether moiety, Dyes Pigm. 98 (2013), 347-357.

[21] I. Kang, J. -Y. Back, R. Kim, Y. -H. Kim, S. -K. Kwon, High efficient and high color pure blue light emitting materials: new asymmetrically highly twisted host and guest based on anthracene, Dyes Pigm. 92 (2012), 588-595. 
[22] S. Shaligram, P. P. Wadgaonkar, U. K. Kharul, Fluorescent polymeric ionic liquids for the detection of nitroaromatic explosives, J. Mater. Chem. A 2 (2014), 13983-13989.

[23] B. Xu, J. He, Y. Liu, B. Xu, Q. Zhu, M. Xie, Z. Zheng, Z. Chi, W. Tian, C. Jin, F. Zhao, Y. Zhang, J. Xu, High-performance two-photon absorption luminophores: large action cross sections, free from fluorescence quenching and tunable emission of efficient non-doped organic light-emitting diodes, J. Mater. Chem. C 2 (2014), 3416-3428.

[24] International Union of Pure and Applied Chemistry, Pure and Applied Chemistry, 45 (1976), p. 99.

[25] T. Liu, K. Zhao, K. Liu, L. Ding, S. Yin, Y. Fang, Synthesis, optical properties and explosive sensing performances of a series of novel $\pi$-conjugated aromatic end-capped oligothiophenes, J. Hazard. Mater. 246-247 (2013), 52-60.

[26] M. Dong, Y. -W. Wang, A. -J. Zhang, Y. Peng, Colorimetric and fluorescent chemosensors for the detection of 2, 4, 6-trinitrophenol and investigation of their co-crystal structures, Chem. Asian J. 8 (2013), 1321-1330.

[27] Y. Ma, H. Li, S. Peng, L. Wang, Highly Selective and Sensitive Fluorescent Paper Sensor for Nitroaromatic Explosive Detection, Anal. Chem. 84 (2012), 8415-8421.

[28] Y. Wang, Y. Ni, Molybdenum disulfide quantum dots as a photoluminescence sensing platform for 2, 4, 6-trinitrophenol detection, Anal. Chem. 86 (2014), 7463-7470.

[29] X. Deng, D. Wu, Highly sensitive photoluminescence energy transfer detection for 2, 4, 6-trinitrophenol using photoluminescent carbon nanodots, RSC Adv. 4 (2014), 42066-42070.

[30] Y. Zheng, M. Miao, M. C. Kemei, R. Seshadri, F. Wudl, The pyreno-triazinyl radicalmagnetic and sensor properties, Isr. J. Chem. 54 (2014), 774-778.

[31] S. Kaur, A. Gupta, V. Bhalla, M. Kumar, Pentacenequinone derivatives: aggregationinduced emission enhancement, mechanism and fluorescent aggregates for superamplified detection of nitroaromatic explosives, J. Mater. Chem. C 2 (2014), 7356-7363. 
[32] O. Pinrat, K. Boonkitpatarakul, W. Paisuwan, M. Sukwattanasinitt, A. Ajavakom, Glucopyranosyl-1, 4-dihydropyridine as a new fluorescent chemosensor for selective detection of 2, 4, 6-trinitrophenol, Analyst 140 (2015), 1886-1893.

[33] A. Pandith, A. Kumar, J. -Y. Lee, H. -S. Kim, 9-Anthracenecarboxamide fluorescent probes for selective discrimination of picric acid from mono- and di-nitrophenols in ethanol, Tetrahedron Lett. 56 (2015), 7094-7099.

[34] P. Vishnoi, S. Sen, G. N. Patwari, R. Murugavel, Charge transfer aided selective sensing and capture of picric acid by triphenylbenzenes, New J. Chem. 39 (2015), 886-892. 
(a)

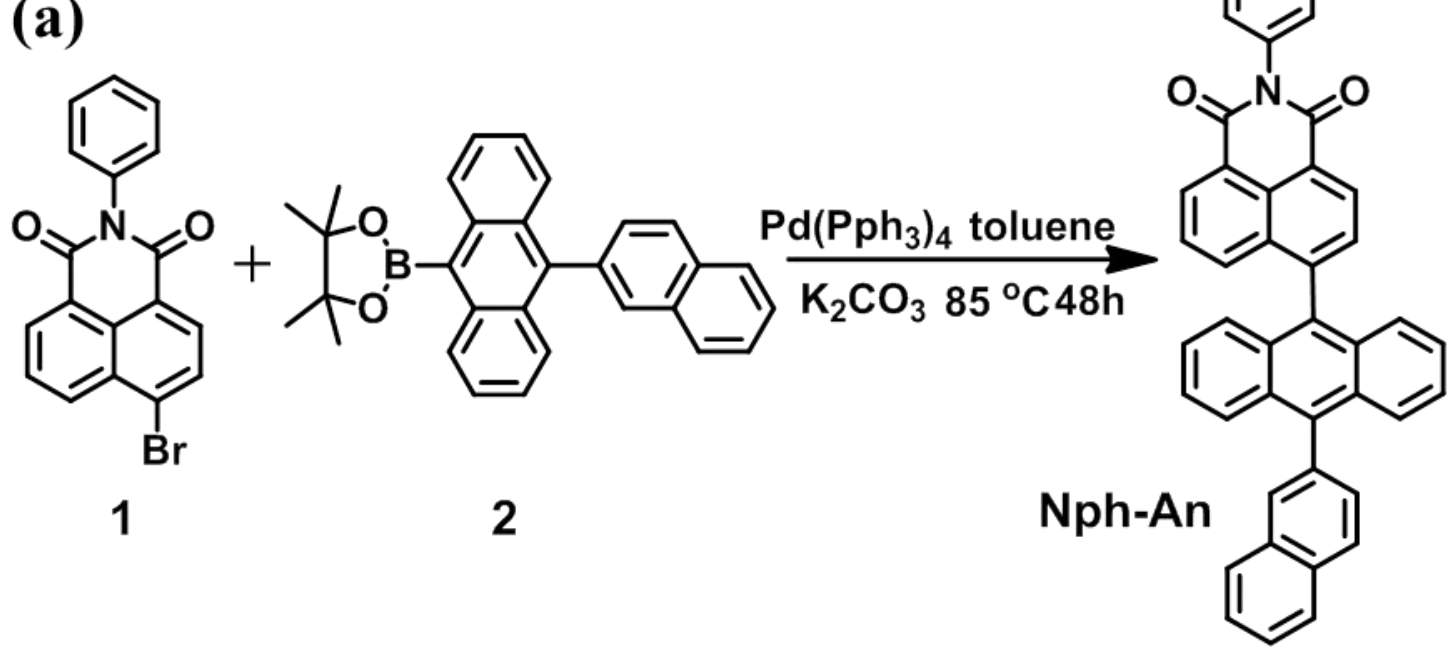

(b)

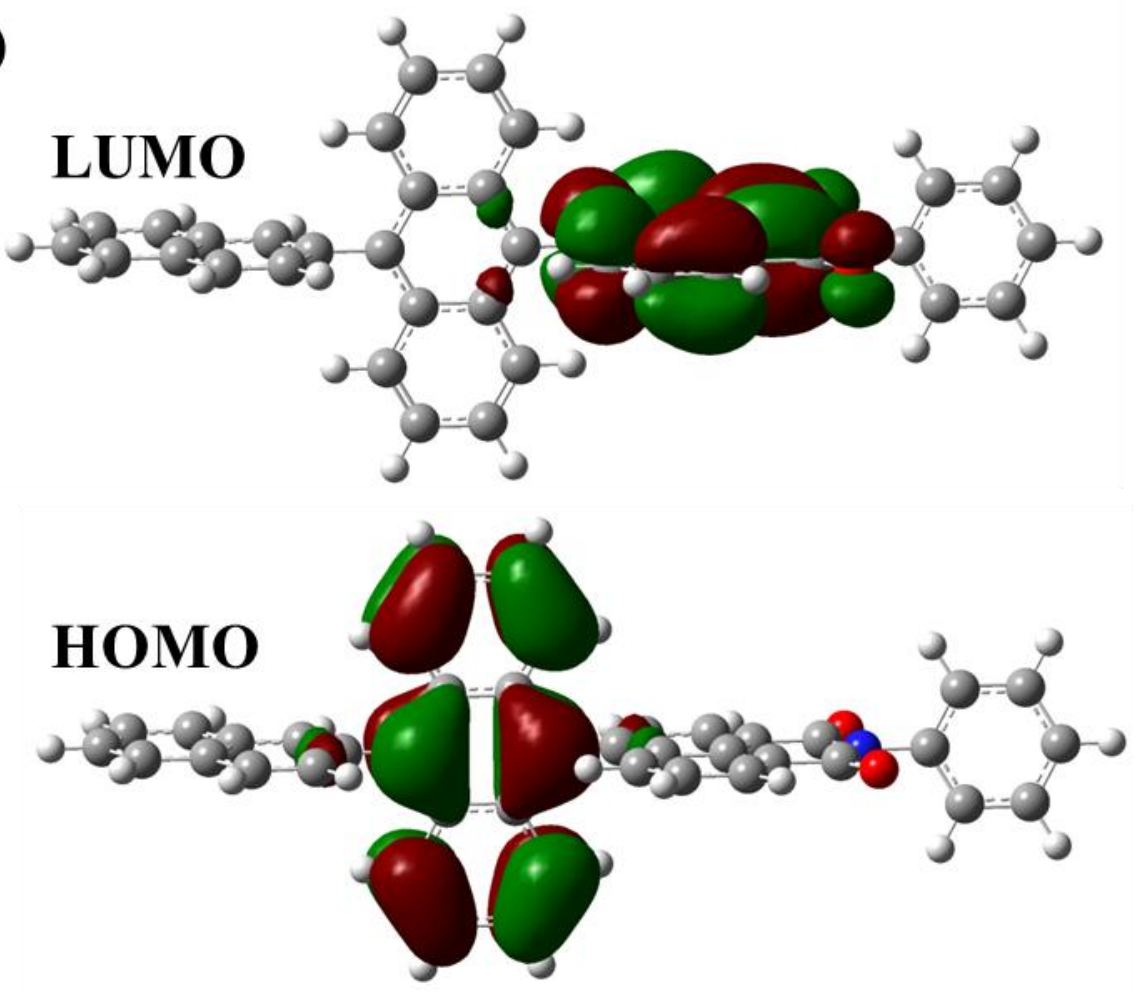



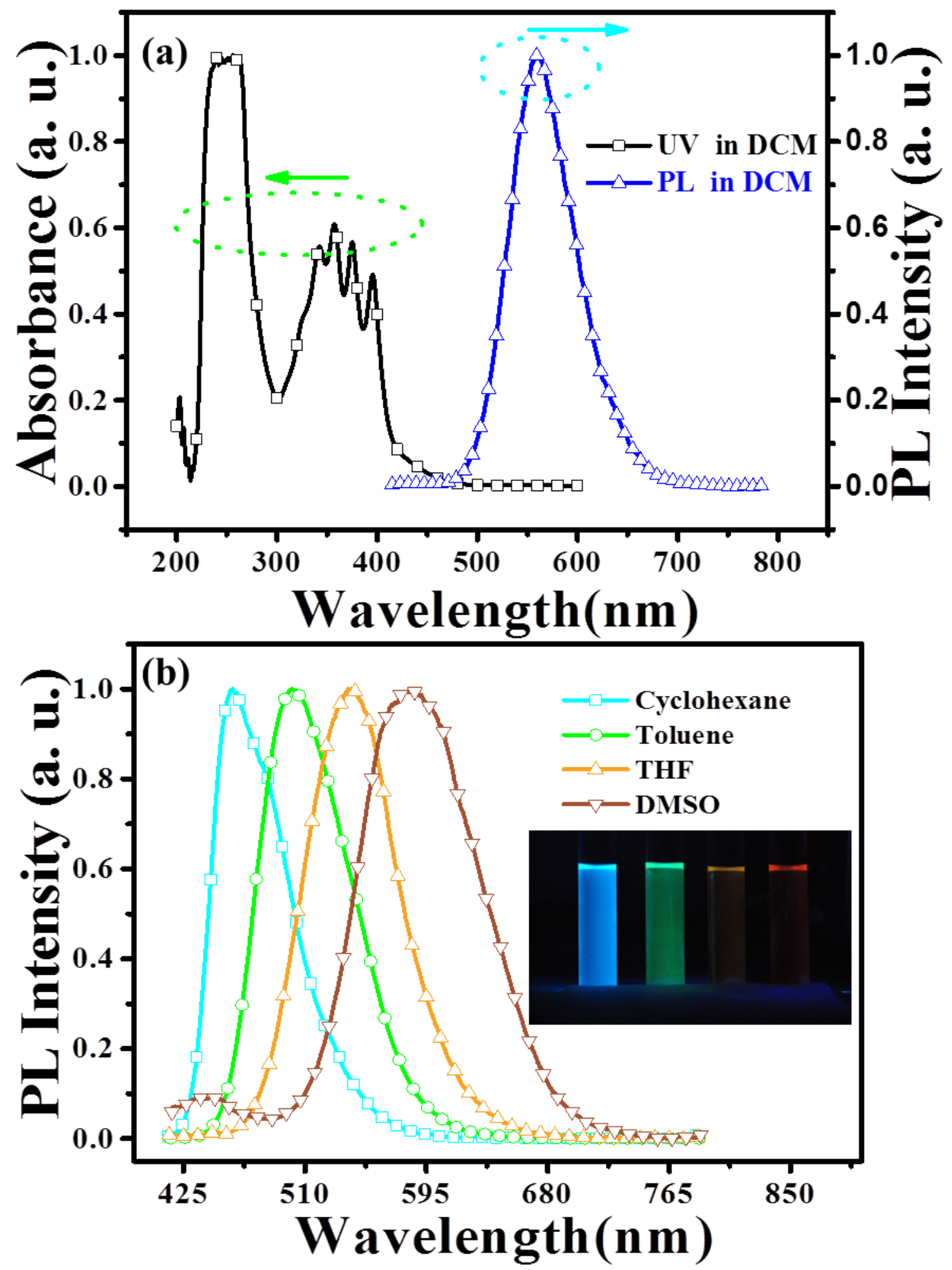


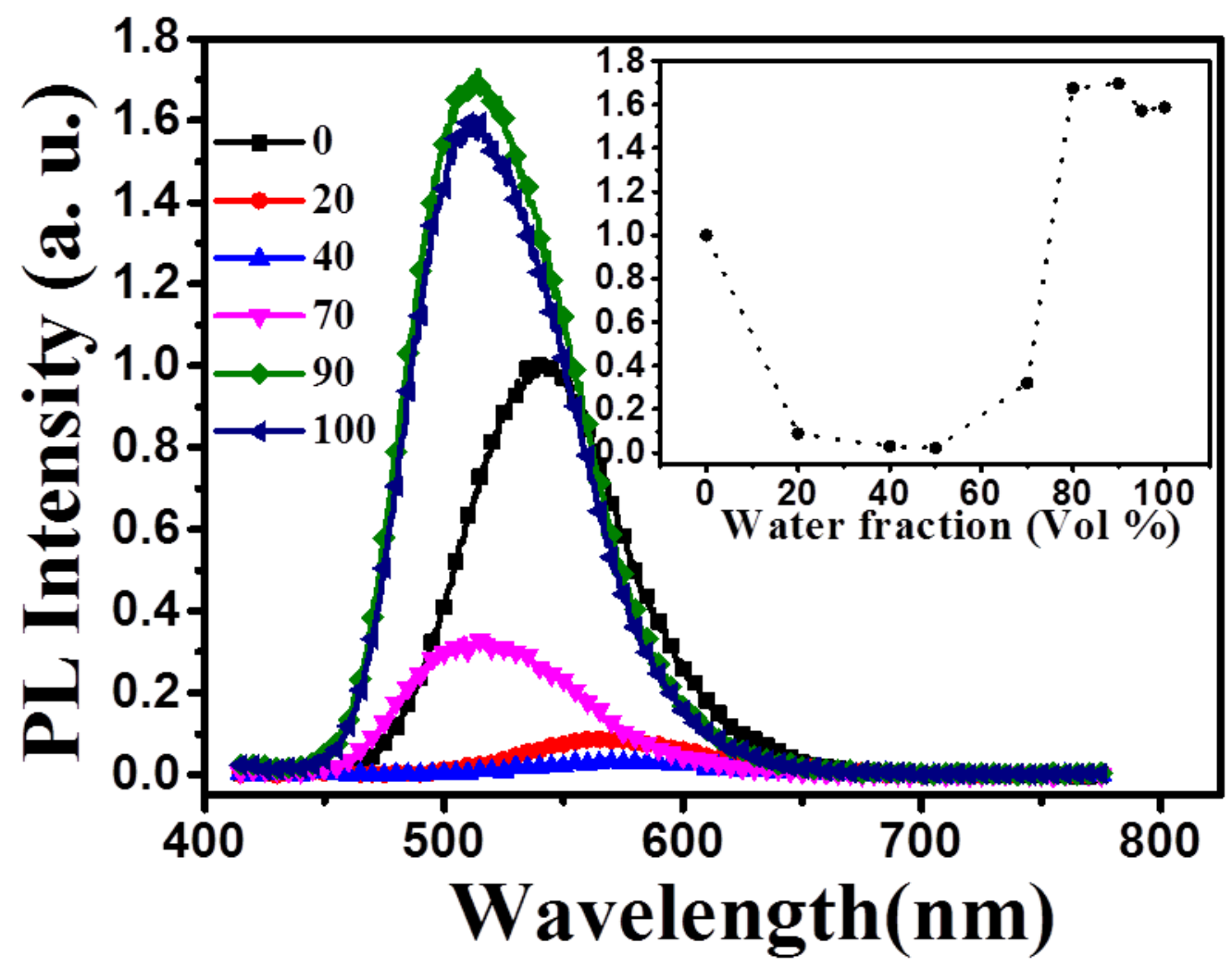



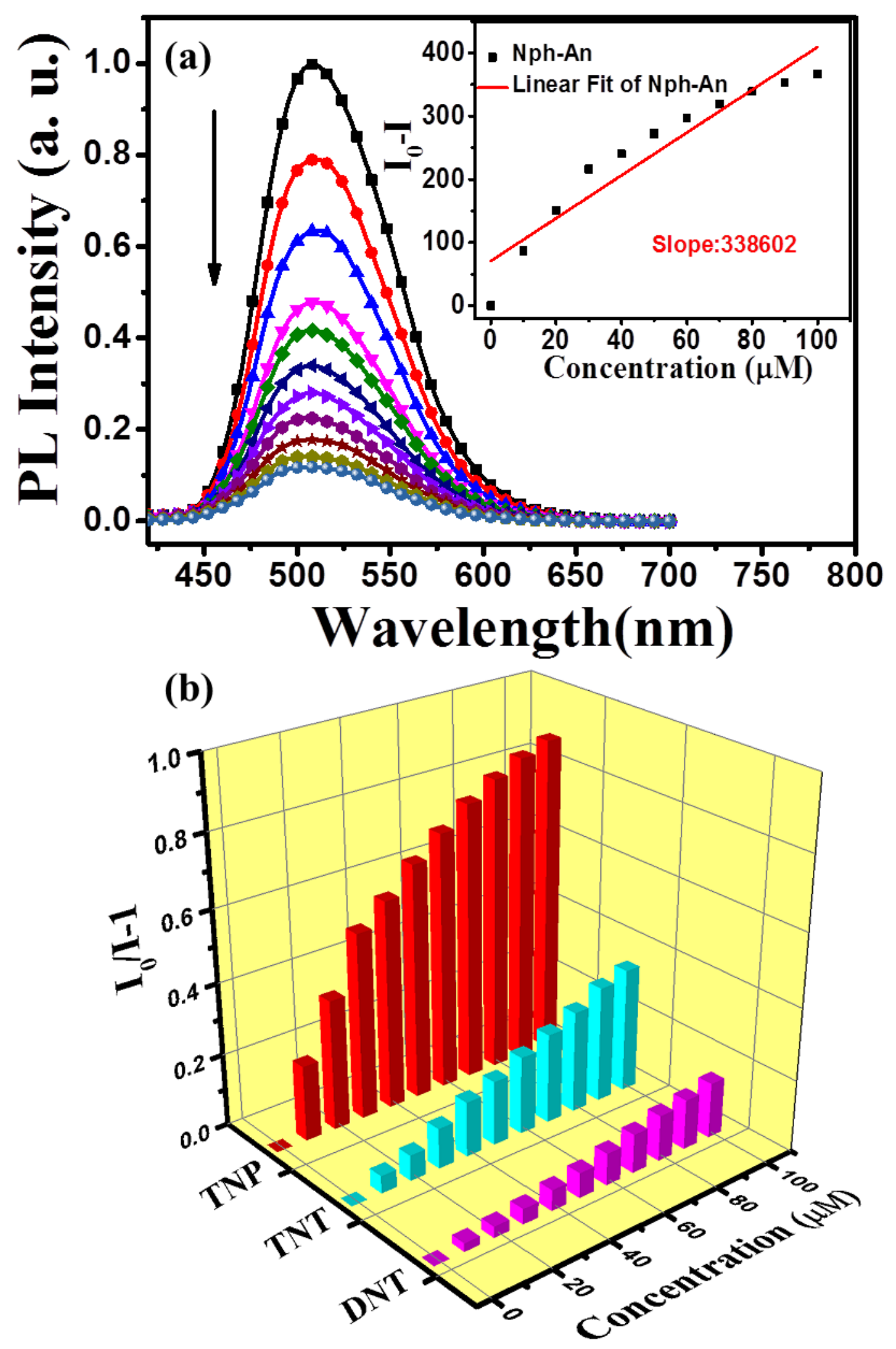

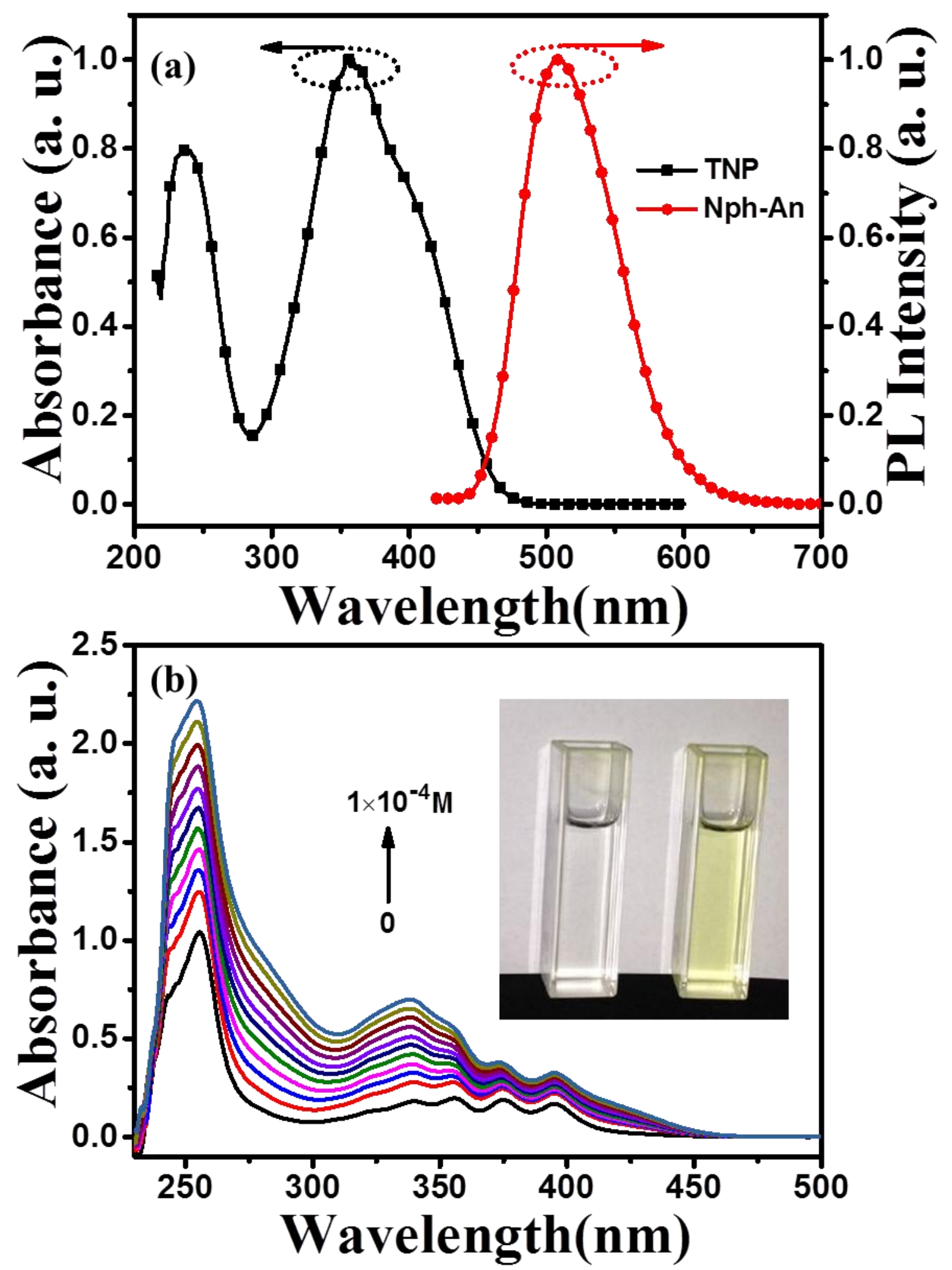


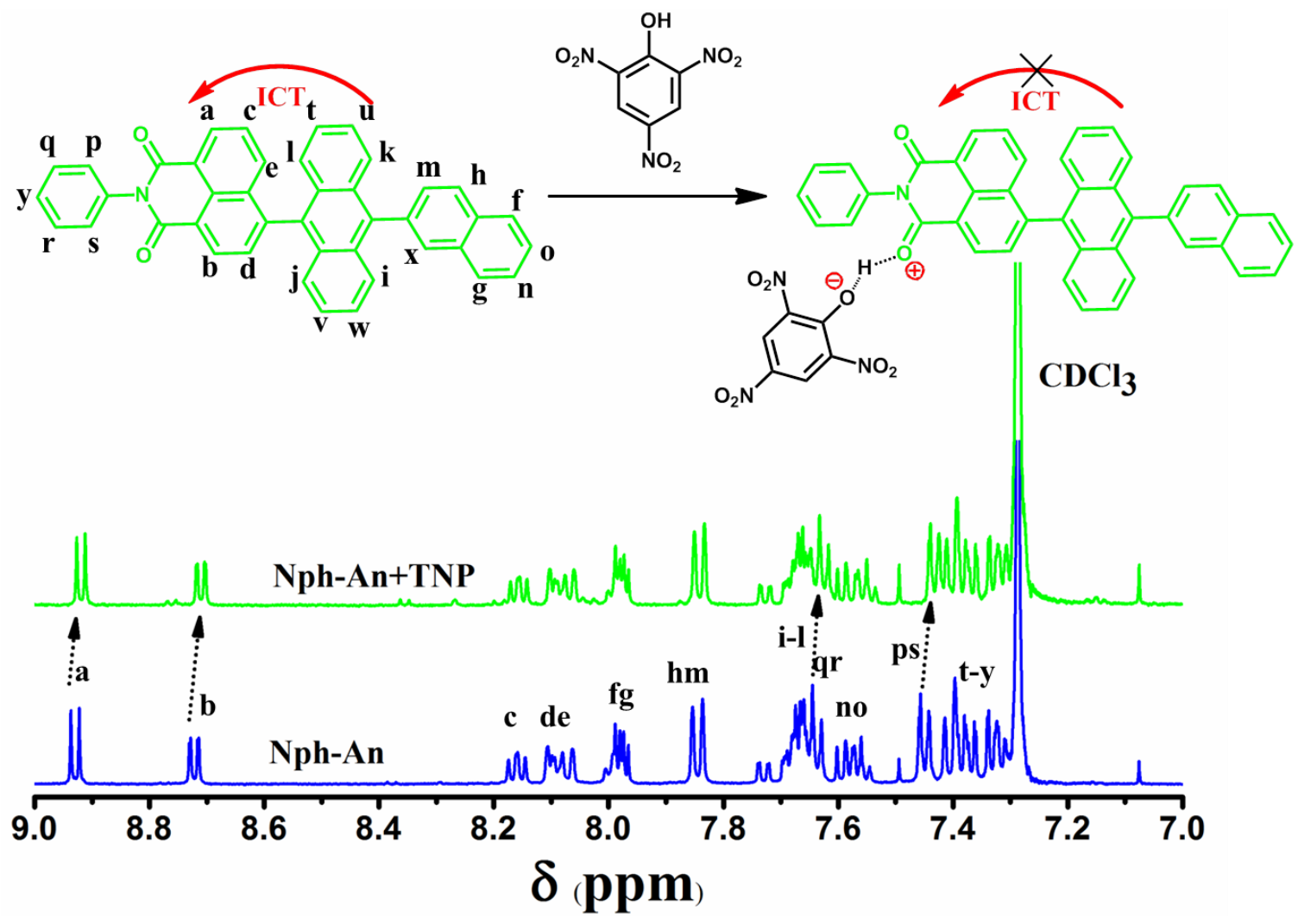




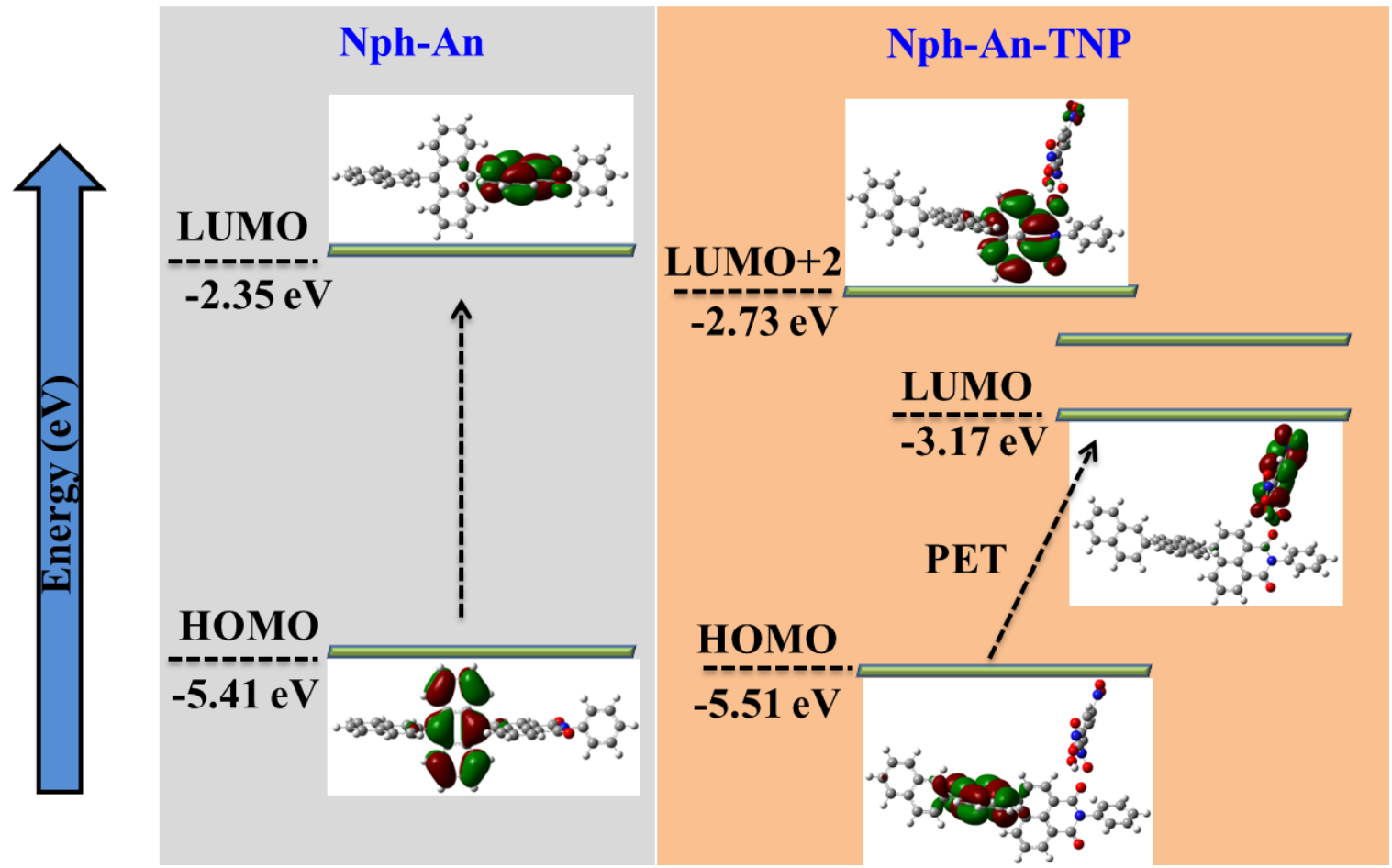



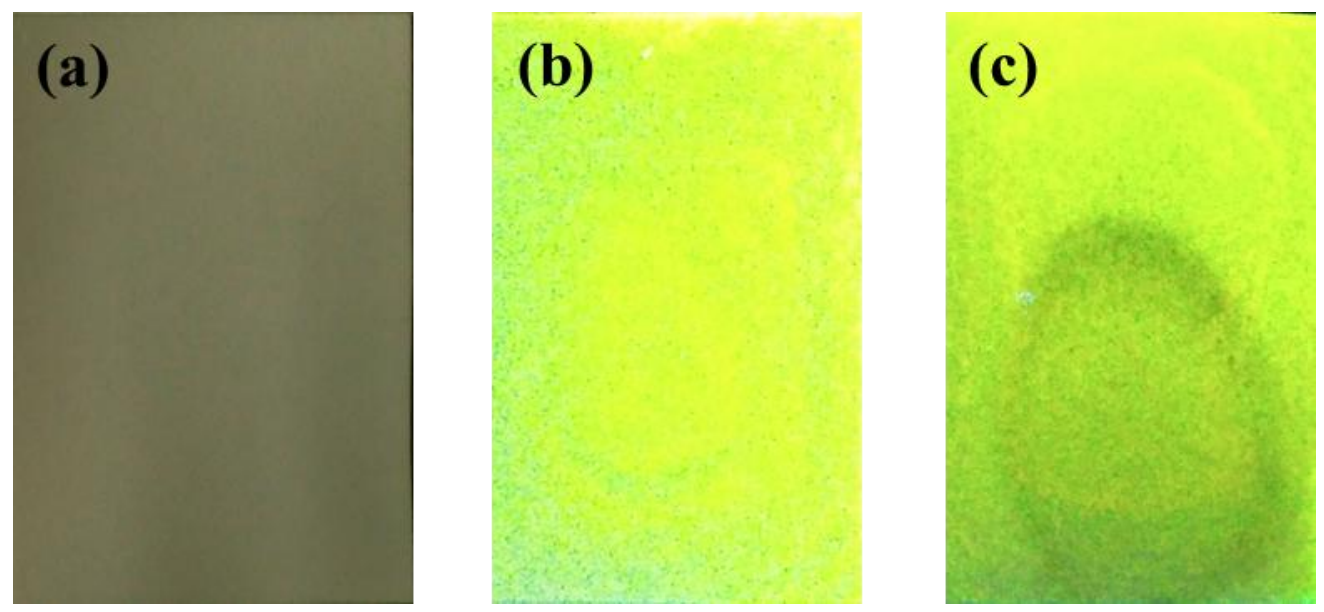


\begin{tabular}{|c|c|c|c|}
\hline Sensing materials & Limit of detection & Solvent & Reference \\
\hline $\mathbf{L}$ & $4.30 \times 10^{-7} \mathrm{M}$ & DMF & [4] \\
\hline 1 & $2.59 \times 10^{-6} \mathrm{M}$ & DMSO & [9] \\
\hline DNSA-SQ & $0.70 \times 10^{-7} \mathrm{M}$ & $\mathrm{CH}_{3} \mathrm{CN}$ & [17] \\
\hline ABA & $2.85 \times 10^{-7} \mathrm{M}$ & THF-Water & [18] \\
\hline 4 & $6.21 \times 10^{-7} \mathrm{M}$ & Ethanol & [25] \\
\hline 1 & $1.08 \times 10^{-7} \mathrm{M}$ & DMF & [26] \\
\hline Alq3 & $1.40 \times 10^{-7} \mathrm{M}$ & Mixed-Water & [27] \\
\hline $\mathrm{MoS}_{2}$ QDs & $0.95 \times 10^{-7} \mathrm{M}$ & Tris-HCl & [28] \\
\hline C-dots & $0.22 \times 10^{-7} \mathrm{M}$ & Phosphate Buffer & [29] \\
\hline PyT & $5.00 \times 10^{-6} \mathrm{M}$ & $\mathrm{CH}_{3} \mathrm{CN}$ & [30] \\
\hline 8 & $2.50 \times 10^{-7} \mathrm{M}$ & DMSO-Water & [31] \\
\hline Glc-DHP & $0.94 \times 10^{-6} \mathrm{M}$ & Water & [32] \\
\hline 1 & $1.00 \times 10^{-6} \mathrm{M}$ & Ethanol & [33] \\
\hline
\end{tabular}

